\title{
Testing for causality in the presence of leading variables
}

\author{
Theologos Pantelidis* \\ Department of Economics, University of Macedonia, Greece
}

Received: 16 October 2014

Revised: 20 December 2014

Accepted: 23 December 2014

\begin{abstract}
This paper provides useful guidelines to practitioners who investigate causality-in-mean and/or causality-in-variance within a trivariate system by means of the two-step procedure proposed by Cheung and $\mathrm{Ng}$ (Journal of Econometrics, 1996) and modified by Hong (Journal of Econometrics, 2001). Specifically, this study highlights cases that can mislead the researcher into reporting false causal relations among the variables under scrutiny. The results of Monte Carlo simulations reveal the seriousness of the problem. Finally, an empirical application that investigates causality-in-mean among three major European stock markets illustrates the proper procedure to follow for correct inference.
\end{abstract}

Keywords: causality-in-mean, causality-in-variance, leading variable, simulation JEL Classification Codes: C15, C22

\section{Introduction}

The investigation of causal relations between financial variables is crucial for most participants in the international money markets. Granger (1969) introduced the concept of Granger causality between two (groups of) variables. In general, a variable Granger causes another variable when past information of the former is necessary to obtain optimal forecasts for the latter. Since the seminal work of Granger (1969), a number of alternative methodologies have been developed to test for Granger causality within a system of variables. Most of these methodologies are designed to examine the existence of either causality-inmean or causality-in-variance.

One of the Granger causality tests available in the literature is the two-step procedure proposed by Cheung and $\mathrm{Ng}$ (1996) and modified by Hong (2001). The proposed test statistic

\footnotetext{
*E-mail: pantelidis@uom.gr.
}

Citation: Pantelidis, T. (2015) Testing for causality in the presence of leading variables, Economics and Business Letters, 4(1), 17-29. 
has two important characteristics that make it attractive to practitioners ${ }^{1}$. First, it is based on simple univariate models and thus it avoids the estimation of complicated multivariate models. Second, it can be used to test for both causality-in-mean and causality-in-variance. On the other hand, this causality test is (by construction) suitable for bivariate system of variables.

The test statistic can be used in the context of a system of more than two variables. However, in this case researchers should be careful when interpreting the results of the test to avoid reaching false conclusions. Misleading results are likely to arise in the presence of a "leading" variable that Granger causes two variables of the system. In order to clarify this point, assume a system of three variables, say $\left(x_{1 t}, x_{2 t}, x_{3 t}\right)$, where $x_{3 t}$ Granger causes both $x_{1 t}$ and $x_{2 t}$, while there is no causality between $x_{1 t}$ and $x_{2 t}$. In this case, it is likely that the two-step causality test will erroneously indicate the existence of bidirectional causality between $x_{1 t}$ and $x_{2 t}$. A different case that can also mislead the researcher to false conclusions arises when $x_{3 t}$ Granger causes only $x_{2 t}$, while there is contemporaneous correlation between $x_{3 t}$ and $x_{1 t}$. In this case, the causality test might erroneously indicate that $x_{1 t}$ Granger causes $x_{2 t}$. In both scenarios, the problem arises from the fact that the two-step causality test is based on (estimated) univariate models that ignore the effect of $x_{3 t}$ on $x_{1 t}$ and $x_{2 t}$. Thus, one of the main advantages of the test, that is the estimation of simple univariate models instead of multivariate models, can become a serious disadvantage. Fortunately, there exists a simple way to overcome this problem. The solution requires that the effect of $x_{3 t}$ on $x_{1 t}$ and $x_{2 t}$ is filtered out when modeling $x_{1 t}$ and $x_{2 t}$.

This paper first uses a simple theoretical example to highlight the problem and explain the dynamics that cause the two-step causality test to indicate the existence of false causal relations when implemented to a trivariate system. Afterwards, by means of Monte Carlo simulations, this study attempts to identify the seriousness of the problem. Moreover, the study proposes a simple way to ensure that the reported (by the test) causality between two series is correct. Finally, the proper procedure to follow for correct inference is illustrated in an empirical application that investigates causality-in-mean among three major European stock markets. It is important to note that this study does not address the issue of omitted variables nor it questions the good performance of the causality test of Cheung and $\mathrm{Ng}$ (1996) and Hong (2001).

The rest of the paper is organised as follows. Section 2 briefly describes the causality test proposed by Cheung and $\mathrm{Ng}$ (1996) and modified by Hong (2001). Section 3 describes a theoretical example to highlight cases that can lead to incorrect conclusions about causality between two variables. The main findings of a Monte Carlo simulation experiment, aiming at examining the size and power properties of the test statistics under consideration, are reported in Section 4. Section 5 presents an empirical example and finally Section 6 concludes the paper.

\section{The two-step causality test}

Cheung and $\mathrm{Ng}$ (1996) introduced a method to test for the existence of Granger causal relations in either the mean or the variance of two series, say $\left(x_{1 t}, x_{2 t}\right)$. The method has two stages. In the first stage, univariate time-series models are estimated for both series under scrutiny, i.e.

\footnotetext{
${ }^{1}$ Among others, Speight and McMillan (2001), Bhar and Hamori (2005) and Inagaki (2007) base their studies on this two-step methodology.
} 


$$
\begin{aligned}
& x_{1 t}=\mu_{1, t}+\sigma_{1, t} e_{1 t} \\
& x_{2 t}=\mu_{2, t}+\sigma_{2, t} e_{2 t}
\end{aligned}
$$

where $e_{1 t}$ and $e_{2 t}$ are two zero mean, independent white noises with unit variances. This specification allows for time variation in both the conditional means and the conditional variances. The univariate ARMA models with GARCH specification in the conditional variance are special cases of this representation.

In the second stage, the researcher can test for (i) causality-in-mean based on the sample cross-correlations of the standardised residuals $\left(\hat{e}_{i t} \equiv \frac{x_{i t}-\mu_{i, t}}{\widehat{\sigma}_{i, t}}, i=1,2\right)$ and/or (ii) causality-invariance based on the sample cross-correlations of the squared standardised residuals $\left(\hat{e}_{i t}^{2}, i=1,2\right)$.

Let $\hat{u}_{i t}, i=1,2$ be either the standardised residuals $\hat{e}_{i t}$ (for the causality-in-mean test) or the squared standardised residuals $\hat{e}_{i t}^{2}$ (for the causality-in-variance test). Then the sample cross-correlation function of $\hat{u}_{1 t}$ and $\hat{u}_{2 t}\left(\hat{\rho}_{1,2}(k)\right)$ is given by the following formulas:

$$
\hat{\rho}_{1,2}(k)=\frac{\hat{C}_{1,2}(k)}{\sqrt{\hat{C}_{1,1}(0) * \hat{C}_{2,2}(0)}}
$$

where

$$
\hat{C}_{1,2}(k)=\left\{\begin{array}{l}
T^{-1} \sum_{t=k+1}^{T}\left[\left(\hat{u}_{1 t}-\bar{u}_{1 t}\right) *\left(\hat{u}_{2 t-k}-\bar{u}_{2 t}\right)\right], k \geq 0 \\
T^{-1} \sum_{t=-k+1}^{T}\left[\left(\hat{u}_{1 t+k}-\bar{u}_{1 t}\right) *\left(\hat{u}_{2 t}-\bar{u}_{2 t}\right)\right], k<0
\end{array}\right.
$$

$T$ is the sample size, $\bar{u}_{1 t}$ is the sample mean of $\hat{u}_{1 t}$ and $\hat{C}_{i, i}(0)$ is the sample variance of $\hat{u}_{i t}, i=1,2$.

The test statistic proposed by Cheung and $\mathrm{Ng}$ (1996) is the following:

$$
S=T \sum_{k=j}^{M} \hat{\rho}_{1,2}^{2}(k)
$$

It can be shown that asymptotically $S$ follows a $X_{M-j+1}^{2}$ distribution ${ }^{2}$. If we set $j=1$ then $S$ can be used to test whether $x_{2 t}$ Granger causes $x_{1 t}$. Under the null hypothesis, there is no Granger causality from $x_{2 t}$ to $x_{1 t}$. On the other hand, we can use $S=T \sum_{k=-M}^{-1} \hat{\rho}_{1,2}^{2}(k)$ to test whether $x_{1 t}$ Granger causes $x_{2 t}$.

Hong (2001) modified the $S$-statistic by introducing a weighting scheme for the sample cross-correlation at each lag. The proposed statistic is the following:

$$
Q_{1}=\frac{T \sum_{j=1}^{T-1} k^{2}\left(\frac{j}{M}\right) * \widehat{\rho}_{1,2}^{2}(j)-C_{1 T}(k)}{\sqrt{2 * D_{1 T}(k)}}
$$

where $C_{1 T}(k)=\sum_{j=1}^{T-1}\left(1-\frac{j}{T}\right) * k^{2}\left(\frac{j}{M}\right), D_{1 T}(k)=\sum_{j=1}^{T-1}\left(1-\frac{j}{T}\right) *\left(1-\frac{j+1}{T}\right) * k^{4}\left(\frac{j}{M}\right)$ and $k(j / M)$ is a weighting function ${ }^{3}$. Under the null hypothesis of no Granger causality and some appropriate regularity conditions, $Q_{1}$ follows asymptotically a $\mathrm{N}(0,1)$ distribution ${ }^{4}$.

\footnotetext{
${ }^{2}$ Small sample versions of the statistic are also available.

${ }^{3}$ When Hong (2001) calculates the cross covariances of the (squared) standardised residuals, he sets $\bar{u}_{1 t}$ and $\bar{u}_{2 t}$ equal to their theoretical value which is zero (unity). A small sample version of the statistic is also available.

${ }^{4} S$ and $Q_{1}$ are one-sided tests and upper-tailed critical values should be used.
} 
In most cases, $Q_{1}$ generates qualitatively similar results irrespectively of the weighting function used. On the other hand, the number of cross-correlations used in the calculation of both aforementioned statistics, determined by $M$, is important especially for S. Cheung and $\mathrm{Ng}$ (1996) argue that $M$ should be large enough to include the largest nonzero sample crosscorrelations. However, a large $M$ is likely to impose a cost in terms of the efficiency of $S$ (as argued by Hong, 2001). The utilization of a weighting function makes $Q_{1}$ less sensitive to the value of $M$. In practice, researchers should check the robustness of their findings across alternative values of $M$. More information about the two statistics can be found in Cheung and $\mathrm{Ng}$ (1996) and Hong (2001) ${ }^{5}$.

\section{A theoretical example}

Consider the following zero-mean and stable VAR(1) model:

$$
\left[\begin{array}{l}
x_{1 t} \\
x_{2 t} \\
x_{3 t}
\end{array}\right]=\left[\begin{array}{ccc}
a_{11} & 0 & a_{13} \\
0 & a_{22} & a_{23} \\
0 & 0 & a_{33}
\end{array}\right]\left[\begin{array}{l}
x_{1 t-1} \\
x_{2 t-1} \\
x_{3 t-1}
\end{array}\right]+\left[\begin{array}{l}
v_{1 t} \\
v_{2 t} \\
v_{3 t}
\end{array}\right]
$$

or equivalently in matrix notation:

$$
X_{t}=A X_{t-1}+V_{t}
$$

where $V_{t}$ is zero-mean white noise process with covariance matrix $\Sigma_{V}$, that is $V_{t} \sim\left(0, \Sigma_{V}\right)\left(\Sigma_{V}\right.$ $\left.:=\left[\sigma_{i j}\right], i, j=1,2,3\right)$ and $E\left(V_{t} V_{s}\right)=0$ when $\mathrm{t} \neq s^{6}$. Assume that a researcher wants to examine the existence of causality-in-mean between the three variables of the model based on the $\mathrm{S}$ (or $Q_{1}$ ) statistic. In the context of this model, there is no causality between $x_{1 t}$ and $x_{2 t}$. In order to investigate causality between $x_{1 t}$ and $x_{2 t}$, the researcher first fits a univariate model to each one of the series, say $x_{i t}=a_{i i} x_{i t-1}+u_{i t}, i=1$, 2. It is straightforward to see that $u_{i t}=a_{i 3} x_{3 t-1}+v_{i t}$, $i=1,2$. Consider the following two cases:

Case $1\left(a_{33} \neq 0\right)$ : It is easy to rewrite $u_{i t}, i=1,2$ as follows:

$$
u_{i t}=v_{i t}+\sum_{k=1}^{\infty} a_{i 3} a_{33}^{k-1} v_{3 t-k}, \quad i=1,2
$$

The cross-correlations between $u_{1 t}$ and $u_{2 t}$ are:

$$
E\left(u_{1 t} u_{2 t-p}\right)=\left\{\begin{array}{c}
a_{23} a_{33}^{p-1} \sigma_{13}+a_{13} a_{23} \sigma_{33} \sum_{k=0}^{\infty} a_{33}^{p+2 k} \text { if } p<0 \\
\sigma_{12}+a_{13} a_{23} \sigma_{33} \sum_{k=0}^{\infty} a_{33}^{2 k} \text { if } p=0 \\
a_{13} a_{33}^{p-1} \sigma_{23}+a_{13} a_{23} \sigma_{33} \sum_{k=0}^{\infty} a_{33}^{p+2 k} \text { if } p>0
\end{array}\right.
$$

Case $2\left(a_{33}=0\right)$ : It is easy to rewrite $u_{i t}, i=1,2$ as follows:

\footnotetext{
${ }^{5}$ See also Van Dijk et al. (2005) for an examination of the effect of structural breaks in volatility on the size properties of the two-step test statistics.

${ }^{6}$ In order to avoid unnecessary complications, this example assumes homoscedastic innovations.
} 


$$
u_{i t}=v_{i t}+a_{i 3} v_{3 t-1}, \quad i=1,2
$$

The cross-correlations between $u_{1 t}$ and $u_{2 t}$ are:

$$
E\left(u_{1 t} u_{2 t-p}\right)= \begin{cases}\sigma_{12}+a_{13} a_{23} \sigma_{33} & \text { if } p=0 \\ a_{13} \sigma_{23} & \text { if } p=1 \\ a_{23} \sigma_{13} & \text { if } p=-1 \\ 0 & \text { if }|p|>1\end{cases}
$$

It is clear that in both cases the cross-correlations between $u_{1 t}$ and $u_{2 t \mp p}$ are in general nonzero although there is no causality between $x_{1 t}$ and $x_{2 t}$. Therefore, the utilization of the $S$ (or $Q_{1}$ ) statistic will erroneously indicate the existence of causality between $x_{1 t}$ and $x_{2 t}$. This happens because the researcher ignored the effect of the "leading" variable, $x_{3 t}$, on both $x_{1 t}$ and $x_{2 t}$ when fitting a model to describe $x_{1 t}$ and $x_{2 t}$. More specifically, when $x_{3 t}$ Granger causes both $x_{1 t}$ and $x_{2 t}$ and $x_{3 t}$ is excluded from the estimated models for $x_{1 t}$ and $x_{2 t}$, the tests will probably indicate the existence of bidirectional causality between $x_{1 t}$ and $x_{2 t}$. This scenario is described in Figure 1 (Panel A). Moreover, the cross-correlations calculated above reveal a different scenario that might lead to false inference. Assume that $x_{3 t}$ Granger causes only (say) $x_{2 t}$ (i.e. $a_{23} \neq 0$ and $a_{13}=0$ ), while there is also contemporaneous correlation between $x_{3 t}$ and $x_{1 t}$ (i.e. $\sigma_{13} \neq 0$ ). In this case, $E\left(u_{1 t} u_{2 t+1}\right)$ is different from zero and thus the test statistics might indicate that $x_{1 t}$ Granger causes $x_{2 t}$. This scenario is described in Panel B of Figure 1. It is obvious that the possibility of having this kind of interactions that can lead to incorrect conclusions increases with the number of variables under scrutiny.

The aforementioned example concentrates on testing for causality-in-mean. It is straightforward to think of an example that highlights the same problem when testing for causality-in-variance by means of the same statistics. Once again, the presence of a "leading" variable can cause similar problems and lead to false conclusions. Fortunately, it is easy to obtain reliable results by simply including lags of the "leading" variable in the estimated models for $x_{1 t}$ and $x_{2 t}$. The researcher should then repeat the causality test to check whether there is any true causality between $x_{1 t}$ and $x_{2 t}$.

\section{Monte Carlo simulations}

The simple theoretical example described in the previous section aimed at highlighting cases that can mislead the researcher into reporting false causal relations among the variables under scrutiny. This section reveals the size of the problem by means of Monte Carlo simulations. The experiment is based on two different Data Generating Processes (DGPs). The first one, $D G P-1$, is used to examine the behavior of the two-step causality tests when testing for causality-in-mean, while the second one, $D G P-2$, is used to examine the behavior of the statistics when testing for causality-in-variance. In general, the experiment examines three different cases for the sample size $T(T=500,1000$ and 2000 observations).

\subsection{Causality-in-mean}

Assume a system of three variables, say $\left(x_{1 t}, x_{2 t}, x_{3 t}\right)$. The system is assumed to be complete, i.e. there are no omitted variables. The first Monte Carlo experiment uses the following DGP to generate random samples: 


$$
D G P-1:\left[\begin{array}{l}
x_{1 t} \\
x_{2 t} \\
x_{3 t}
\end{array}\right]=\left[\begin{array}{l}
0.1 \\
0.1 \\
0.1
\end{array}\right]+\left[\begin{array}{ccc}
0.5 & 0 & a_{13} \\
0 & 0.5 & a_{23} \\
0 & 0 & a_{33}
\end{array}\right]\left[\begin{array}{l}
x_{1 t-1} \\
x_{2 t-1} \\
x_{3 t-1}
\end{array}\right]+\left[\begin{array}{l}
e_{1 t} \\
e_{2 t} \\
e_{3 t}
\end{array}\right]
$$

where

$$
\begin{aligned}
& e_{i t}=\sqrt{h_{i t}} Z_{i t}, i=1,2,3 \\
& h_{i t}=0.001+0.6 h_{i t-1}+0.3 e_{i t-1}^{2}
\end{aligned}
$$

$D G P-1$ describes a VAR(1)-GARCH(1,1) process. It is easy to see that under DGP - 1 , if $a_{13} \neq 0$ and $a_{23} \neq 0, x_{3 t}$ causes (in mean) both $x_{1 t}$ and $x_{2 t}$, while there is no causality between $x_{1 t}$ and $x_{2 t}$. Thus, $x_{3 t}$ plays the role of the "leading" variable in this experiment. Finally, under $D G P$ - 1 there is no causality-in-variance between the three variables.

A set of different values for the parameters of $D G P-1$ is considered. The experiment is based on 2000 replications for each case under examination. In each replication both statistics $(S$ and $Q_{1}$ ) are considered to examine all the different bilateral causalities between the three variables of the system. The results, reported in Tables $1-3^{7}$, can be summarised as follows:

1. The two statistics behave qualitatively similar (see Tables 1 and 2 for the $Q_{1}$ and $S$ statistic respectively).

2. When examining the causal relations between $x_{3 t}$ and either $x_{1 t}$ or $x_{2 t}$, the performance of the statistics is very good. More specifically, the power of the statistics to indicate that $x_{3 t}$ causes both $x_{1 t}$ and $x_{2 t}$ is $100 \%$ in almost all the cases considered in this experiment, while when testing for causality from either $x_{1 t}$ or $x_{2 t}$ to $x_{3 t}$ the empirical size of the statistics is close to the nominal one of $5 \%$. We should note however that $Q_{1}$ is slightly oversized while $\mathrm{S}$ is slightly undersized ${ }^{8}$.

3. Both statistics suffer from severe size distortions when testing for causality between $x_{1 t}$ and $x_{2 t}$. Even when the causal effect of $x_{3 t}$ on the other two variables of the system is relatively weak (i.e. $a_{13}=a_{23}=0.2$ ), $Q_{1}$ rejects the true null hypothesis of no causality from $x_{2 t}$ to $x_{1 t}$ in $12 \%$ of the cases $(\mathrm{T}=500)$. As expected, the size distortions of the statistics increase substantially as the causal effect of $x_{3 t}$ on the other two variables of the system becomes stronger (i.e. as $a_{13}$ and/or $a_{23}$ increase). Moreover, the size distortions of the statistics are higher for larger samples. For example, the size of $Q_{1}$ increases from $55.2 \%$ to $83 \%$ when the sample size increase from 500 to 1000 observations (when $a_{13}=a_{23}=0.5$ ). Finally, the size distortions of the statistics increase with the persistence of the "leading" variable (see Table 3 ). For example, when $T=500, Q_{1}$ erroneously indicates causality from $x_{2 t}$ to $x_{1 t}$ in $15 \%$, $55.2 \%$ and $92.9 \%$ of the cases for $\mathrm{a}_{33}$ equal to $0.2,0.5$ and 0.8 respectively.

Fortunately, the severe size distortions of the two statistics can easily be cured by including the lag of the "leading" variable, $x_{3 t}$, in the estimated models for $x_{1 t}$ and $x_{2 t}$. As soon as $x_{3 t}$ is included in the univariate specification of both $x_{1 t}$ and $x_{2 t}$, the effect of $x_{3 t}$ on the other variables is filtered out and the empirical size of the statistics is close to the nominal one of $5 \%$ (see Table 4).

\footnotetext{
${ }^{7}$ For brevity, the tables report the results for selected cases. Critical values for the $S$ and $Q_{l}$ statistic are obtained from the $X^{2}$ and standard Normal distribution respectively.

${ }^{8}$ We would like to thank an anonymous referee for pointing this out to us.
} 


\subsection{Causality-in-variance}

The second experiment is properly designed to examine causality-in-variance. DGP - 2 considers a system of three variables, say $\left(x_{1 t}, x_{2 t}, x_{3 t}\right)$, which is assumed to be complete, i.e. there are no omitted variables. $D G P-2$ is as follows ${ }^{9}$ :

$$
\begin{aligned}
& D G P-2: x_{i t}=0.1+e_{i t}, \quad i=1,2,3 \\
& e_{i t}=h_{i t} z_{i t}, \quad z_{i t} \sim N(0,1) \\
& h_{1 t}^{2}=0.1+b_{1} e_{1 t-1}^{2}+d_{1} e_{3 t-1}^{2} \\
& h_{2 t}^{2}=0.1+b_{2} e_{2 t-1}^{2}+d_{2} e_{3 t-1}^{2} \\
& h_{3 t}^{2}=0.1+b_{3} e_{3 t-1}^{2}
\end{aligned}
$$

Under DGP - 2, $x_{3 t}$ causes (in variance) both $x_{1 t}$ and $x_{2 t}$ (given that $d_{1} \neq 0$ and $d_{2} \neq 0$ ), while there is no causality between $x_{1 t}$ and $x_{2 t}$. A set of different values for the parameters of $D G P$ 2 is considered. For brevity, Tables 5 and 6 (Panel A) reports the results for $Q_{1}$ and $S$ for only three of the cases examined. The results of alternative specifications are qualitatively similar. The findings are in general similar to $D G P-1$ and can be summarised as follows:

1. When examining the causal relations between $x_{3 t}$ and either $x_{1 t}$ or $x_{2 t}$, the size and power properties of the statistics are very good. Specifically, the power of the test to indicate that $x_{3 t}$ causes both $x_{1 t}$ and $x_{2 t}$ is almost $100 \%$, while the empirical size of the test (when examining the effect of $x_{1 t}$ or $x_{2 t}$ on $x_{3 t}$ ) is close to the nominal one of $5 \%$ (both statistics are marginally oversized).

2. The tests suffers from severe size distortions when testing for causality between $x_{1 t}$ and $x_{2 t}$. The size distortions are slightly higher for $Q_{1}$. The behavior of both statistics deteriorates as the sample size increases. Moreover, the size distortions of $Q_{1}$ and $S$ increase substantially as the causal effect of $x_{3 t}$ on the other two variables of the system becomes stronger (i.e. as $d_{1}$ and/or $d_{2}$ increase).

3. Similar to DGP - 1, the size distortions of the statistics disappear (see Panel B of Table 5 and 6) as soon as the effect of $x_{3 t}$ on both $x_{1 t}$ and $x_{2 t}$ is filtered out by including sufficient lags of $e_{3 t}^{2}$ in the conditional variance specification of $x_{1 t}$ and $x_{2 t}$.

In summary, the results of the simulations show that when examining a trivariate system with the presence of a "leading" variable ( say $x_{3 t}$ ) that Granger causes the other two variables (say $x_{1 t}$ and $x_{2 t}$ ), the $S$ and $Q_{1}$ statistics suffer from severe size distortions. More specifically, the two test statistics have the tendency to erroneously indicate the existence of bidirectional causality between $x_{1 t}$ and $x_{2 t}$. A simple way to overcome this problem is to filter out the effect of $x_{3 t}$ on $x_{1 t}$ and $x_{2 t}$ in the univariate specification of $x_{1 t}$ and $x_{2 t}$.

The implication of the findings of this study for empirical applications is as follows: whenever a researcher investigates the existence of causality-in-mean or causality-in-variance within a system of three variables based on either $S$ or $Q_{1}$, she has to be very cautious when interpreting the results. To be more specific, this study suggests that whenever the test statistics indicate (i) bidirectional causality between two variables (say $x_{1 t}$ and $x_{2 t}$ ) and (ii) the existence of a third variable (say $x_{3 t}$ ) that Granger causes both $x_{1 t}$ and $x_{2 t}$, the researcher must proceed in further investigation of the relation between $x_{1 t}$ and $x_{2 t}$. In order to verify that causality between these two variables is correct, the researcher must repeat the causality tests by filtering out the effect of $x_{3 t}$ on $x_{1 t}$ and $x_{2 t}$ in their univariate specification.

\footnotetext{
${ }^{9}$ Similarly, alternative distributions, such as the $t$-distribution, could be considered for $z_{i t}$.
} 


\section{An empirical example}

This section presents an empirical example that illustrates the proper procedure to follow for correct inference when performing a causality analysis based on either the $S$ or the $Q_{1}$ test. The application examines causality-in-mean among the stock market returns of three major European countries, namely Austria, France and Germany. The analysis is based on daily stock market returns calculated as the logarithmic difference of the corresponding stock market index ${ }^{10}$. The period under investigation spans from $04 / 01 / 2000$ to $28 / 11 / 2014$ resulting in a total of 3,702 observations.

Initially, an $\mathrm{AR}(\mathrm{p})-\mathrm{GARCH}(1,1)$ model is fitted in each series where $\mathrm{p}$ is large enough to capture the autocorrelation pattern of the series. In the second stage of the analysis, the $Q_{1}$ statistic is calculated based on the standardized residuals of the three estimated models to examine the existence of causality-in-mean between the markets under scrutiny. The results, reported in Panel A of Table 7, reveal a number of causal relations. The results suggest that Germany causes both Austria and France. Keeping in mind the results of the simulations reported in the previous section, we should check whether the reported causality between Austria and France is deceptive and caused by the effect of Germany on these two markets. It turns out that this is the case. Panel B of Table 7 reports the results of the causality tests when a lag of the German stock return is included in the models of Austria and France. It is clear that the test finds no signs of causality between Austria and France.

The empirical analysis described in this section is a simple example of a situation where the researcher can be misled to false conclusions if she ignores the point raised in this study, that is, in the context of trivariate system of variables, the existence of a "leading" variable that Granger causes the other two variables of the system can (if not taken into account) mislead the two-step causality test to erroneously indicate the existence of false causal relations.

\section{Conclusions}

This study highlights cases that can mislead the two-step procedure proposed by Cheung and $\mathrm{Ng}$ (1996) and modified by Hong (2001) into reporting false causal relations among the variables of interest. More specifically, the study focuses on the size and power properties of the two causality tests in the context of trivariate systems. Initially, a theoretical example illustrates cases that can mislead the test statistics into reporting false causal relations among the variables under examination. This can happen if the system under scrutiny contains a "leading" variable ( say $x_{3 t}$ ) that Granger causes two of the variables of the system (say $x_{1 t}$ and $x_{2 t}$ ), while there in no causality between $x_{1 t}$ and $x_{2 t}$. In such a case, the test statistics have the tendency to erroneously indicate the existence of bidirectional causality between $x_{1 t}$ and $x_{2 t}$. Afterwards, a set of Monte Carlo simulations reveal the size of the problem. The test statistics seem to suffer from severe size distortions that, interestingly, increase with the sample size.

These findings have important implications for empirical works. This paper highlights the need for careful examination of empirical results before reaching final conclusions. Fortunately, in a trivariate system, there is a simple way to find out whether the reported causality between two variables is true or false. The researcher should simply filter out the effect of the "leading" variable when modeling $x_{1 t}$ and $x_{2 t}$.

\footnotetext{
${ }^{10}$ Data source: Yahoo Finance.
} 


\section{References}

Bhar, R. and Hamori, S. (2005) Causality in variance and the type of traders in crude oil futures, Energy Economics, 27(3), 527-539.

Cheung, Y.M. and Ng, L.K. (1996) A causality-in-variance test and its application to financial market prices, Journal of Econometrics, 72(1-2), 33-48.

Granger, C.W.J. (1969) Investigating causal relations by econometric models and crossspectral methods, Econometrica, 37(3), 424-438.

Hong, Y. (2001) A test for volatility spillover with application to exchange rates, Journal of Econometrics, 103(1-2), 183-224.

Inagaki, K. (2007) Testing for volatility spillover between the British pound and the euro, Research in International Business and Finance, 21(2), 161-174.

Speight, A.E.H and McMillan, D.G. (2001) Volatility spillovers in East European blackmarket exchange rates, Journal of International Money and Finance, 20(3), 367-378.

Van Dijk, D., Osborn, D.R. and Sensier, M. (2005) Testing for causality in variance in the presence of breaks, Economics Letters, 89(2), 193-199. 
Table 1. Percent rejections of $H_{0}$ : "No causality" $\left(Q_{1} \text {-statistic, DGP-1, } \alpha_{33}=0.5\right)^{*}$

\begin{tabular}{|c|c|c|c|c|c|c|}
\hline \multirow[b]{3}{*}{$\alpha_{13}=\alpha_{23}=0.2$} & \multicolumn{6}{|c|}{$\mathrm{T}=500$} \\
\hline & $\mathrm{X}_{2 \mathrm{t}} \rightarrow \mathrm{X}_{1 \mathrm{t}}$ & $X_{1 t} \rightarrow X_{2 t}$ & $X_{3 t} \rightarrow X_{1 t}$ & $X_{1 t} \rightarrow X_{3 t}$ & $X_{3 t} \rightarrow X_{2 t}$ & $\mathrm{X}_{2 \mathrm{t}} \rightarrow \mathrm{X}_{3 \mathrm{t}}$ \\
\hline & 12.0 & 10.5 & 99.5 & 7.2 & 99.7 & 6.3 \\
\hline$\alpha_{13}=\alpha_{23}=0.5$ & 55.2 & 52.9 & 100.0 & 6.2 & 100.0 & 5.9 \\
\hline \multirow[t]{3}{*}{$\alpha_{13}=\alpha_{23}=0.8$} & 81.8 & 81.0 & 100.0 & 5.9 & 100.0 & 5.6 \\
\hline & \multicolumn{6}{|c|}{$T=1000$} \\
\hline & $X_{2 t} \rightarrow X_{1 t}$ & $X_{1 t} \rightarrow X_{2 t}$ & $X_{3 t} \rightarrow X_{1 t}$ & $X_{1 t} \rightarrow X_{3 t}$ & $X_{3 t} \rightarrow X_{2 t}$ & $X_{2 t} \rightarrow X_{3 t}$ \\
\hline$\alpha_{13}=\alpha_{23}=0.2$ & 17.3 & 17.3 & 100.0 & 6.9 & 100.0 & 6.6 \\
\hline$\alpha_{13}=\alpha_{23}=0.5$ & 83.0 & 82.7 & 100.0 & 6.7 & 100.0 & 5.8 \\
\hline \multirow[t]{3}{*}{$\alpha_{13}=\alpha_{23}=0.8$} & 98.5 & 98.0 & 100.0 & 5.5 & 100.0 & 5.3 \\
\hline & \multicolumn{6}{|c|}{$\mathrm{T}=2000$} \\
\hline & $\mathrm{X}_{2 \mathrm{t}} \rightarrow \mathrm{X}_{1 \mathrm{t}}$ & $X_{1 t} \rightarrow X_{2 t}$ & $X_{3 t} \rightarrow X_{1 t}$ & $X_{1 t} \rightarrow X_{3 t}$ & $X_{3 t} \rightarrow X_{2 t}$ & $X_{2 t} \rightarrow X_{3 t}$ \\
\hline$\alpha_{13}=\alpha_{23}=0.2$ & 26.9 & 26.8 & 100.0 & 6.5 & 100.0 & 7.0 \\
\hline$\alpha_{13}=\alpha_{23}=0.5$ & 98.2 & 98.9 & 100.0 & 6.2 & 100.0 & 6.6 \\
\hline$\alpha_{13}=\alpha_{23}=0.8$ & 100.0 & 100.0 & 100.0 & 5.9 & 100.0 & 5.9 \\
\hline
\end{tabular}

Table 2. Percent rejections of $H_{0}$ : "No causality" (S-statistic, DGP-1, $\left.\alpha_{33}=0.5\right) *$

\begin{tabular}{|c|c|c|c|c|c|c|}
\hline \multirow[b]{3}{*}{$\alpha_{13}=\alpha_{23}=0.2$} & \multicolumn{6}{|c|}{$\mathrm{T}=500$} \\
\hline & $X_{2 t} \rightarrow X_{1 t}$ & $X_{1 t} \rightarrow X_{2 t}$ & $X_{3 t} \rightarrow X_{1 t}$ & $X_{1 t} \rightarrow X_{3 t}$ & $X_{3 t} \rightarrow X_{2 t}$ & $X_{2 t} \rightarrow X_{3 t}$ \\
\hline & 6.8 & 6.5 & 97.8 & 5.1 & 98.5 & 4.9 \\
\hline$\alpha_{13}=\alpha_{23}=0.5$ & 31.9 & 30.7 & 100.0 & 4.9 & 100.0 & 4.0 \\
\hline \multirow[t]{3}{*}{$\alpha_{13}=\alpha_{23}=0.8$} & 58.2 & 57.0 & 100.0 & 5.1 & 100.0 & 3.8 \\
\hline & \multicolumn{6}{|c|}{$\mathrm{T}=1000$} \\
\hline & $X_{2 t} \rightarrow X_{1 t}$ & $X_{1 t} \rightarrow X_{2 t}$ & $X_{3 t} \rightarrow X_{1 t}$ & $X_{1 t} \rightarrow X_{3 t}$ & $X_{3 t} \rightarrow X_{2 t}$ & $\mathrm{X}_{2 \mathrm{t}} \rightarrow \mathrm{X}_{3 \mathrm{t}}$ \\
\hline$\alpha_{13}=\alpha_{23}=0.2$ & 9.3 & 9.3 & 100.0 & 5.3 & 100.0 & 4.4 \\
\hline$\alpha_{13}=\alpha_{23}=0.5$ & 62.8 & 62.1 & 100.0 & 4.7 & 100.0 & 4.5 \\
\hline \multirow[t]{3}{*}{$\alpha_{13}=\alpha_{23}=0.8$} & 90.6 & 89.8 & 100.0 & 4.2 & 100.0 & 4.6 \\
\hline & \multicolumn{6}{|c|}{$\mathrm{T}=2000$} \\
\hline & $X_{2 t} \rightarrow X_{1 t}$ & $X_{1 t} \rightarrow X_{2 t}$ & $X_{3 t} \rightarrow X_{1 t}$ & $X_{1 t} \rightarrow X_{3 t}$ & $X_{3 t} \rightarrow X_{2 t}$ & $X_{2 t} \rightarrow X_{3 t}$ \\
\hline$\alpha_{13}=\alpha_{23}=0.2$ & 14.5 & 15.2 & 100.0 & 4.5 & 100.0 & 4.4 \\
\hline$\alpha_{13}=\alpha_{23}=0.5$ & 92.0 & 92.7 & 100.0 & 4.4 & 100.0 & 4.2 \\
\hline$\alpha_{13}=\alpha_{23}=0.8$ & 100.0 & 100.0 & 100.0 & 4.9 & 100.0 & 4.3 \\
\hline
\end{tabular}

Table 3. Percent rejections of $H_{0}$ : "No causality" ( $Q_{1}$-statistic, DGP-1, $\left.\alpha_{13}=\alpha_{23}=0.5\right) *$

\begin{tabular}{|c|c|c|c|c|c|}
\hline \multicolumn{2}{|c|}{$\alpha_{33}=0.2$} & \multicolumn{2}{|c|}{$\mathrm{T}=500$} & \multicolumn{2}{|c|}{$\alpha_{33}=0.8$} \\
\hline $\begin{array}{c}X_{2 t} \rightarrow X_{1 t} \\
15.0\end{array}$ & $\begin{array}{c}X_{1 t} \rightarrow X_{2 t} \\
12.7\end{array}$ & $\underset{55.2}{X_{2 t} \rightarrow X_{1 t}}$ & $\begin{array}{c}X_{1 t} \rightarrow X_{2 t} \\
52.9\end{array}$ & $\begin{array}{c}X_{2 t} \rightarrow X_{1 t} \\
92.9\end{array}$ & $\begin{array}{c}X_{1 \mathrm{t}} \rightarrow X_{2 \mathrm{t}} \\
\quad 92.7\end{array}$ \\
\hline \multicolumn{2}{|c|}{$\alpha_{33}=0.2$} & \multicolumn{2}{|c|}{$\alpha_{33}=0.5$} & \multicolumn{2}{|c|}{$\alpha_{33}=0.8$} \\
\hline $\begin{array}{c}X_{2 t} \rightarrow X_{1 t} \\
21.8\end{array}$ & $\begin{array}{c}\mathrm{X}_{1 \mathrm{t}} \rightarrow \mathrm{X}_{2 \mathrm{t}} \\
21.5\end{array}$ & $\begin{array}{c}\mathrm{X}_{2 \mathrm{t}} \rightarrow \mathrm{X}_{1 \mathrm{t}} \\
83.0\end{array}$ & $\begin{array}{c}X_{1 \mathrm{t}} \rightarrow \mathrm{X}_{2 \mathrm{t}} \\
82.7\end{array}$ & $\begin{array}{c}X_{2 t} \rightarrow X_{1 \mathrm{t}} \\
99.9\end{array}$ & $\begin{array}{c}X_{1 \mathrm{t}} \rightarrow X_{2 \mathrm{t}} \\
100.0\end{array}$ \\
\hline \multicolumn{2}{|c|}{$\alpha_{33}=0.2$} & \multicolumn{2}{|c|}{$\begin{array}{l}T=2000 \\
\alpha_{33}=0.5\end{array}$} & \multicolumn{2}{|c|}{$\alpha_{33}=0.8$} \\
\hline $\begin{array}{c}X_{2 t} \rightarrow X_{1 t} \\
35.4\end{array}$ & $\begin{array}{c}X_{1 \mathrm{t}} \rightarrow X_{2 \mathrm{t}} \\
\quad 35.6\end{array}$ & $\begin{array}{c}X_{2 t} \rightarrow X_{1 t} \\
\quad 98.2\end{array}$ & $\begin{array}{c}X_{1 \mathrm{t}} \rightarrow \mathrm{X}_{2 \mathrm{t}} \\
\quad 98.9\end{array}$ & $\begin{array}{c}X_{2 t} \rightarrow X_{1 t} \\
100.0\end{array}$ & $\begin{array}{c}X_{1 \mathrm{t}} \rightarrow X_{2 \mathrm{t}} \\
100.0\end{array}$ \\
\hline
\end{tabular}

\footnotetext{
* "a $\rightarrow \mathrm{b}$ " stands for one-way causality from a to $\mathrm{b}$.
} 
Table 4. Percent rejections of $H_{0}$ : "No causality" (DGP-1, $\left.\alpha_{13}=\alpha_{23}=\alpha_{33}=0.5\right) *$ after removing the effect of the "leading” variable

\begin{tabular}{ccc|cc}
\hline & \multicolumn{2}{c}{$\mathrm{S}$} & \multicolumn{2}{c}{$\mathrm{Q}_{1}$} \\
& $\mathrm{X}_{2 \mathrm{t}} \rightarrow \mathrm{X}_{1 \mathrm{t}}$ & $\mathrm{X}_{1 \mathrm{t}} \rightarrow \mathrm{X}_{2 \mathrm{t}}$ & $\mathrm{X}_{2 \mathrm{t}} \rightarrow \mathrm{X}_{1 \mathrm{t}}$ & $\mathrm{X}_{1 \mathrm{t}} \rightarrow \mathrm{X}_{2 \mathrm{t}}$ \\
$\mathrm{T}=500$ & 4.9 & 4.6 & 6.7 & 6.8 \\
$\mathrm{~T}=1000$ & 4.4 & 5.0 & 7.7 & 6.5 \\
$\mathrm{~T}=2000$ & 4.5 & 4.1 & 6.9 & 6.4 \\
\hline \hline
\end{tabular}

Table 5. Percent rejections of $H_{0}$ : "No causality" $\left(Q_{1} \text {-statistic, DGP-2, } b_{1}=b_{2}=b_{3}=0.8\right)^{*}$

\begin{tabular}{|c|c|c|c|c|c|c|}
\hline \multirow{6}{*}{$\begin{array}{l}\mathrm{d}_{1}=\mathrm{d}_{2}=0.3 \\
\mathrm{~d}_{1}=\mathrm{d}_{2}=0.6 \\
\mathrm{~d}_{1}=\mathrm{d}_{2}=0.9\end{array}$} & \multicolumn{6}{|c|}{ Panel A (effect of "leading" variable is ignored) } \\
\hline & \multicolumn{6}{|c|}{$\mathrm{T}=500$} \\
\hline & $X_{2 t} \rightarrow X_{1 t}$ & $X_{1 t} \rightarrow X_{2 t}$ & $X_{3 t} \rightarrow X_{1 t}$ & $X_{1 t} \rightarrow X_{3 t}$ & $\mathrm{X}_{3 \mathrm{t}} \rightarrow \mathrm{X}_{2 \mathrm{t}}$ & $\mathrm{X}_{2 \mathrm{t}} \rightarrow \mathrm{X}_{3 \mathrm{t}}$ \\
\hline & 31.2 & 32.0 & 99.1 & 6.9 & 98.2 & 6.1 \\
\hline & 37.5 & 37.6 & 99.9 & 6.7 & 99.9 & 5.9 \\
\hline & 38.9 & 40.1 & 99.9 & 6.7 & 99.9 & 5.5 \\
\hline & \multicolumn{6}{|c|}{$\mathrm{T}=1000$} \\
\hline & $X_{2 t} \rightarrow X_{1 t}$ & $X_{1 t} \rightarrow X_{2 t}$ & $X_{3 t} \rightarrow X_{1 t}$ & $X_{1 t} \rightarrow X_{3 t}$ & $X_{3 t} \rightarrow X_{2 t}$ & $\mathrm{X}_{2 \mathrm{t}} \rightarrow \mathrm{X}_{3 \mathrm{t}}$ \\
\hline $\mathrm{d}_{1}=\mathrm{d}_{2}=0.3$ & 44.4 & 43.9 & 100.0 & 5.7 & 99.9 & 6.3 \\
\hline $\mathrm{d}_{1}=\mathrm{d}_{2}=0.6$ & 52.0 & 50.8 & 100.0 & 5.2 & 100.0 & 6.0 \\
\hline \multirow[t]{2}{*}{$\mathrm{d}_{1}=\mathrm{d}_{2}=0.9$} & 54.2 & 53.5 & 100.0 & 5.1 & 100.0 & 6.2 \\
\hline & \multicolumn{6}{|c|}{$\mathrm{T}=2000$} \\
\hline & $X_{2 t} \rightarrow X_{1 t}$ & $X_{1 t} \rightarrow X_{2 t}$ & $X_{3 t} \rightarrow X_{1 t}$ & $X_{1 t} \rightarrow X_{3 t}$ & $X_{3 t} \rightarrow X_{2 t}$ & $X_{2 t} \rightarrow X_{3 t}$ \\
\hline $\mathrm{d}_{1}=\mathrm{d}_{2}=0.3$ & 64.4 & 63.5 & 100.0 & 7.3 & 100.0 & 5.9 \\
\hline $\mathrm{d}_{1}=\mathrm{d}_{2}=0.6$ & 71.6 & 71.1 & 100.0 & 7.5 & 100.0 & 6.0 \\
\hline \multirow[t]{4}{*}{$\mathrm{d}_{1}=\mathrm{d}_{2}=0.9$} & 73.4 & 73.6 & 100.0 & 7.3 & 100.0 & 6.2 \\
\hline & \multicolumn{6}{|c|}{ Panel B (effect of "leading" variable is taken into account) } \\
\hline & \multicolumn{2}{|c|}{$\mathrm{d}_{1}=\mathrm{d}_{2}=0.3$} & \multicolumn{2}{|c|}{$\mathrm{d}_{1}=\mathrm{d}_{2}=0.6$} & \multicolumn{2}{|c|}{$\mathrm{d}_{1}=\mathrm{d}_{2}=0.9$} \\
\hline & $X_{2 t} \rightarrow X_{1 t}$ & $X_{1 t} \rightarrow X_{2 t}$ & $X_{2 t} \rightarrow X_{1 t}$ & $\mathrm{X}_{1 \mathrm{t}} \rightarrow \mathrm{X}_{2 \mathrm{t}}$ & $X_{2 t} \rightarrow X_{1 t}$ & $X_{1 t} \rightarrow X_{2 t}$ \\
\hline $\mathrm{T}=500$ & 6.5 & 6.0 & 6.5 & 6.1 & 6.5 & 6.2 \\
\hline $\mathrm{T}=1000$ & 5.9 & 7.2 & 6.0 & 7.2 & 6.1 & 7.2 \\
\hline $\mathrm{T}=2000$ & 6.4 & 6.7 & 6.3 & 6.7 & 6.3 & 6.7 \\
\hline
\end{tabular}

\footnotetext{
* " $\mathrm{a} \rightarrow \mathrm{b}$ " stands for one-way causality from a to $\mathrm{b}$.
} 
Table 6. Percent rejections of $H_{0}$ : “No causality" (S-statistic, DGP-2, $\left.b_{1}=b_{2}=b_{3}=0.8\right)^{*}$

\begin{tabular}{|c|c|c|c|c|c|c|}
\hline & \multicolumn{6}{|c|}{ Panel A (effect of "leading" variable is ignored) } \\
\hline & \multicolumn{6}{|c|}{$\mathrm{T}=500$} \\
\hline & $\mathrm{X}_{2 \mathrm{t}} \rightarrow \mathrm{X}_{1 \mathrm{t}}$ & $\mathrm{X}_{1 \mathrm{t}} \rightarrow \mathrm{X}_{2 \mathrm{t}}$ & $X_{3 t} \rightarrow X_{1 t}$ & $X_{1 t} \rightarrow X_{3 t}$ & $\mathrm{X}_{3 \mathrm{t}} \rightarrow \mathrm{X}_{2 \mathrm{t}}$ & $X_{2 t} \rightarrow X_{3 t}$ \\
\hline $\mathrm{d}_{1}=\mathrm{d}_{2}=0.3$ & 27.1 & 27.1 & 97.5 & 5.5 & 96.8 & 5.6 \\
\hline $\mathrm{d}_{1}=\mathrm{d}_{2}=0.6$ & 31.4 & 32.1 & 99.8 & 5.6 & 99.6 & 5.8 \\
\hline \multirow[t]{3}{*}{$\mathrm{d}_{1}=\mathrm{d}_{2}=0.9$} & 33.3 & 33.3 & 99.9 & 5.8 & 99.9 & 5.8 \\
\hline & \multicolumn{6}{|c|}{$\mathrm{T}=1000$} \\
\hline & $X_{2 t} \rightarrow X_{1 t}$ & $X_{1 t} \rightarrow X_{2 t}$ & $X_{3 t} \rightarrow X_{1 t}$ & $X_{1 t} \rightarrow X_{3 t}$ & $X_{3 t} \rightarrow X_{2 t}$ & $X_{2 t} \rightarrow X_{3 t}$ \\
\hline $\mathrm{d}_{1}=\mathrm{d}_{2}=0.3$ & 39.5 & 38.5 & 100 & 5.7 & 100 & 5.9 \\
\hline $\mathrm{d}_{1}=\mathrm{d}_{2}=0.6$ & 45.4 & 44.3 & 100 & 6.1 & 100 & 6.7 \\
\hline \multirow[t]{2}{*}{$\mathrm{d}_{1}=\mathrm{d}_{2}=0.9$} & 47.2 & 45.7 & 100 & 5.9 & 100 & 6.6 \\
\hline & \multicolumn{6}{|c|}{$\mathrm{T}=2000$} \\
\hline & $X_{2 t} \rightarrow X_{1 t}$ & $X_{1 t} \rightarrow X_{2 t}$ & $X_{3 t} \rightarrow X_{1 t}$ & $X_{1 t} \rightarrow X_{3 t}$ & $X_{3 t} \rightarrow X_{2 t}$ & $X_{2 t} \rightarrow X_{3 t}$ \\
\hline $\mathrm{d}_{1}=\mathrm{d}_{2}=0.3$ & 56.8 & 55.7 & 100 & 5.6 & 99.9 & 5.7 \\
\hline $\mathrm{d}_{1}=\mathrm{d}_{2}=0.6$ & 63.2 & 62.9 & 100 & 6.6 & 100 & 6.2 \\
\hline \multirow[t]{4}{*}{$\mathrm{d}_{1}=\mathrm{d}_{2}=0.9$} & 65.1 & 65.3 & 100 & 6.5 & 100 & 6.4 \\
\hline & \multicolumn{6}{|c|}{ Panel B (effect of "leading" variable is taken into account) } \\
\hline & \multicolumn{2}{|c|}{$\mathrm{d}_{1}=\mathrm{d}_{2}=0.3$} & \multicolumn{2}{|c|}{$\mathrm{d}_{1}=\mathrm{d}_{2}=0.6$} & \multicolumn{2}{|c|}{$\mathrm{d}_{1}=\mathrm{d}_{2}=0.9$} \\
\hline & $\mathrm{X}_{2 \mathrm{t}} \rightarrow \mathrm{X}_{1 \mathrm{t}}$ & $X_{1 t} \rightarrow X_{2 t}$ & $X_{2 t} \rightarrow X_{1 t}$ & $X_{1 t} \rightarrow X_{2 t}$ & $X_{2 t} \rightarrow X_{1 t}$ & $\mathrm{X}_{1 \mathrm{t}} \rightarrow \mathrm{X}_{2 \mathrm{t}}$ \\
\hline $\mathrm{T}=500$ & 5.3 & 4.3 & 5.2 & 4.4 & 5.4 & 4.6 \\
\hline $\mathrm{T}=1000$ & 5.0 & 5.8 & 5.0 & 5.9 & 4.9 & 6.0 \\
\hline $\mathrm{T}=2000$ & 5.8 & 5.9 & 5.6 & 5.9 & 5.6 & 5.8 \\
\hline
\end{tabular}

Table 7. Causality tests

\begin{tabular}{|c|c|c|c|}
\hline & \multicolumn{3}{|c|}{ Panel A } \\
\hline & AUS & FR & GER \\
\hline AUS causes & --- & -0.738 & -0.910 \\
\hline FR causes & 2.869 & --- & -0.846 \\
\hline \multirow[t]{2}{*}{ GER causes } & 7.043 & $\begin{array}{c}3.338 \\
\text { Panel B }\end{array}$ & --- \\
\hline & AUS & FR & GER \\
\hline AUS causes & --- & -0.836 & -0.915 \\
\hline FR causes & -0.765 & --- & -0.822 \\
\hline GER causes & -0.047 & -0.541 & --- \\
\hline
\end{tabular}

AUS: Austria, FR: France, GER: Germany

Bold numbers indicate rejection of the null hypothesis of no causality at a 5\% confidence level.

\footnotetext{
* " $\mathrm{a} \rightarrow \mathrm{b}$ " stands for one-way causality from a to $\mathrm{b}$.
} 
Figure 1. Graphical representation of possible causal relations among three variables

\begin{tabular}{|c|c|c|c|c|c|c|c|}
\hline Panel & $\left.{ }_{23} \neq 0\right)$ & & & $1 \mathrm{BC}$ & $\neq 0, c$ & $\neq 0)$ & \\
\hline True Process & Test Result & & Ie $\operatorname{Prc}$ & & & st $\mathrm{R}$ & \\
\hline $\mathrm{X}_{1 \mathrm{t}}$ & & $X_{3 t}$ & $\rightarrow$ & $\mathrm{X}_{2 \mathrm{t}}$ & $X_{3 t}$ & $\rightarrow$ & $X_{2 t}$ \\
\hline $\mathrm{X}_{3 \mathrm{t}}$ & & $X_{3 t}$ & A & $\mathrm{X}_{1 \mathrm{t}}$ & $X_{3 t}$ & A & $\mathrm{X}_{1 \mathrm{t}}$ \\
\hline $\boldsymbol{x}_{2 \mathrm{t}}$ & $\Delta_{Y}$ & $\mathrm{X}_{3 \mathrm{t}}$ & \langle\rangle$\left.^{\prime}\right\rangle$ & $\mathrm{X}_{1 \mathrm{t}}$ & & & \\
\hline$X_{1 t}$ & . & $X_{1 t}$ & A & $\mathrm{X}_{2 \mathrm{t}}$ & & $\rightarrow$ & $\mathbf{X}$ \\
\hline
\end{tabular}

"a $\rightarrow$ b" stands for one-way causality from a to $b$.

"a↔b" stands for bilateral causality between a and $b$.

" $\mathrm{a}<\mathrm{T}>\mathrm{b}$ " stands for contemporaneous causality between a and $\mathrm{b}$. 